\title{
FAIRE PARLER L'ABSENCE : UN MODÈLE DE COMMUNICATION CRÉATEUR D'ÉVIDENCE ${ }^{1}$
}

\author{
Emmanuel Belin ${ }^{2}$
}

Les thèmes contemporains du déracinement, du relativisme, du nihilisme apparaissent à beaucoup comme les conséquences lointaines de la sécularisation de l'ordre social contenue dans le projet d'autonomie construit par les Lumières. Ce qu'ils indiquent, c'est que la modernité est un projet exigeant sur le plan psychique, qu'elle implique qu'un deuil soit opéré, par lequel le sujet renonce à toute transparence, toute fondation ultime. Elle requiert un arrachement à ce qu'on appelle parfois la "fonction paternelle", un renoncement à l'idée d'un principe stable et incontestable -qu'on l'appelle "Dieu" ou "Sens de l'histoire" ou encore "Vie". On ne compte plus les publications qui rendent compte de ce désarroi de l'homme moderne face à cette nécessité de repenser son rapport au monde et au sens en termes non substantiels.

1 Ce texte a été rédigé à l'issue d'une observation participante de trois mois (une fois par semaine) dans un groupe de prière, en Belgique francophone, se réclamant du Renouveau Charismatique. Que tous les membres de ce groupe puissent être ici remerciés de l'accueil chaleureux qu'ils m'ont réservé et de leur collaboration bienveillante.

2 Aspirant au Fonds National de la Recherche Scientifique (FNRS). 
Face à ces retournements difficiles à opérer, un discours conservateur prônant le retour à des évidences plus substantielles se répand, alimenté de nostalgie et d'angoisse, mais également d'un désir de dépassement des logiques institutionnelles anonymes -la bureaucratie, le marché, l'ordre mondial- qui garantissent l'ordre en l'absence d'un principe métasocial et dont le caractère systématique, détaché du monde vécu, est ressenti comme une perte de contrôle de l'histoire par les hommes qui la font. Ces appels à la tradition considèrent insupportable l'idée d'une absence de fondement, posant implicitement que la modernité n'est "pas faite pour l'homme", qu'elle ne tient pas compte de ses besoins psychiques ${ }^{1}$. Pour cette raison, les rhétoriques conservatrices. se définissent souvent comme post-matérialistes et reposent sur une mise en avant des carences identitaires liées à une réduction du désir humain au statut de simple envie d'un objet ${ }^{2}$. Toutes les théories post-matérialistes ne sont cependant pas conservatrices: un pan entier de la pensée contemporaine tente, en effet, en repensant les concepts même d'identité et de fondement, d'opérer une "métamorphose de la fonction paternelle", qui soit se traduit par une positivation du doute et du mouvement, posant ceux-ci comme socles identitaires paradoxaux, soit aboutit à une réhabilitation de la raison par des processus de clôture logique comme principe autofondateur. Pour faire court, on peut classer dans la première catégorie les théories post-modernes d'inspiration nietzschéenne, et dans la seconde des théories "ultra-modernes" cherchant à dépasser la modernité dans la modernité. A l'inverse de ces deux courants, le post-matérialisme conservateur refuse de remettre en question les formes consacrées d'identité et de fondement, prônant plutôt une restauration de la tradition et de la foi par-delà la raison. Le regain de religiosité auquel nous assistons actuellement peut dans cette optique être analysé comme la partie émergée d'un iceberg sur lequel la modernité pourrait s'échouer ${ }^{3}$. Il importe par conséquent de saisir en détail le type de rapport à l'évidence qui se joue dans les communautés qui s'en réclament. C'est l'objectif de ce texte.

1 Cf. par exemple l'analyse de Peter SLOTERdijk, "Weimar et la Californie. Note sur la crise de la philosophie de l'histoire et sur la prolifération des doctrines holistiques", Critique, nº464-465, 1986, p. 114-127.

2 Maurice Bellet, La seconde humanité, Paris, Desclée de Brouwer, 1993, p. 40 à 81 entre autres.

3 On peut lire à ce sujet Elisabeth VOLCKRICK, "Le monde sans projet et le retour des oracles", in Emmanuel BELIN et Guy BAJOIT (Eds), Le sujet, à paraître. 
Le point de départ du raisonnement que nous proposons est que les formes de religiosité qui se développent autour de nous ne peuvent pas reposer sur les mêmes mécanismes de fidélisation que celles qui ont précédé les Lumières, parce que le contexte dans lequel elles se développent n'est pas le même. Le caractère évident de la présence et de l'action divine doit aujourd'hui être instauré et produit par ceux-là même qui se proposent d'y souscrire. Des structures extrêmement puissantes doivent assurer l'émergence de certitudes dans un contexte dominé par le doute. Il ne suffit pas, en effet, de désirer croire, encore faut-il y parvenir. L'hypothèse qui sera développée ici est qu'il est possible de mettre en place une mise en scène de la révélation qui satisfasse toutes les conditions d'une conversion. Cette mise en scène est axée autour d'un renversement du maximum de vraisemblance analogue à celui qu'opèrent les prestidigitateurs: tirant parti du désir de croire, il s'y agit de mettre en place une structure intersubjective de production du discours à la fois extrêmement ordonnatrice et totalement invisible, de manière à ce que l'ordre produit apparaisse comme une surprise, dont le caractère involontaire est associé à un statut d'extériorité et de transcendance.

La portée de cette réflexion dépasse la simple anecdote. A travers les mécanismes d'instauration de l'évidence collectivement partagée, c'est à la production de l'institution que nous nous intéressons. C'est qu'en effet, la vie quotidienne est jalonnée d'évidences dont le statut s'apparente à celles qu'instituent les groupes religieux dont il est ici question. Mettre en lumière ces processus, c'est se donner les moyens de comprendre plus en profondeur la possibilité d'une hétéronomie pragmatique, quotidienne, au sein d'un modèle culturel principiellement autonome.

\section{Réflexions déontologiques préalables}

Avant de développer notre raisonnement sur ce point, arrêtonsnous un instant sur quelques questions déontologiques ou éthiques. Il est de bon ton, dans un discours de sciences humaines, de prendre les mesures nécessaires à ce qu'il respecte les sujets et les croyances auxquelles il se réfère. Dans cette optique, un des axiomes fondamentaux de la sociologie et de l'anthropologie de la religion est de ne pas prendre position sur les contenus en termes de véracité, mais seulement d'expliquer les mécanismes sociaux par lesquels sont transmis, 
diffusés ces contenus. Cette approche est louable, certes, mais difficilement applicable dans son intégralité, dès lors que le contenu du discours entre en contradiction explicite, non avec la théorie particulière dont il est l'objet, mais avec ce qui fait la substance profonde du langage scientifique. En d'autres termes, montrer que les contenus religieux peuvent être rapportés à des phénomènes non référés à quelque instance surnaturelle, c'est déjà tenir un discours engagé quand ces contenus affirment ne pouvoir être expliqués que par recours au surnaturel. On l'aura compris, ce problème se pose dans toute son acuité dans la présente analyse. Il introduit l'idée d'un enchâssement entre un discours et un métadiscours, c'est-à-dire un discours portant sur le discours. Quand un langage religieux amalgame les deux niveaux, tout discours autre que religieux sur le discours religieux est blasphématoire et irrespectueux de son objet.

Il semble cependant possible de reposer le dilemme en relativisant la portée même du discours scientifique. Dès qu'on pose celui-ci comme production culturelle de type hypothético-déductif, proposant un type de langage parmi d'autres possibles sur le réel, répondant à un type de questions, il devient légitime d'adopter un ton dubitatif par rapport à certains aspects réflexifs du discours analysé1. En simple, l'analyse proposée respecte les convictions des sujets auxquels elle se rapporte dans la mesure où elle se pose comme une explication alternative, comme un point de vue possible. Elle ne les respecte toutefois pas dans la mesure où, par le fait même de sa formulation, elle contredit un axiome universalisant par un autre jugement de valeur universalisant, affirmant la tolérance et l'acceptation de l'autre.

\section{Projet du texte}

Laissons là le problème éthique insoluble de l'intolérance envers la tolérance, pour construire un schéma possible de la communication

1 “(...) on doit savoir dès le départ que les modes de compréhension des deux parties, la collectivité et ceux qui l'étudient, sont les uns et les autres filtrés par une idéologie, par une culture. Ensuite, on remarque que ce que retient le filtre des uns, celui des autres le laisse passer, que ce que masque la culture des premiers se manifeste dans celle des seconds. Ceci suffirait à fonder pour les uns et pour les autres la nécessité de la démarche anthropologique, entre autres manières de confrontation. (Albert DouTrelouX et Colette DeGive, "Perspective anthropologique sur un mouvement religieux actuel", in Social Compass, XXV, 1978/1, p. 45 . 
transcendantale qui ne fasse pas appel au surnaturel. Ce schéma, basé sur l'empathie et l'intuition phénoménologique, n'est encore qu'une hypothèse gauche. Il prend, avons-nous dit, pour point de départ la nécessité du dialogue dans l'expérience intense de la révélation. L'enjeu sera donc de développer un mode de pensée qui puisse faire d'un locuteur le spectateur de ses propres productions intellectuelles, comportementales, verbales et représentationnelles. Il s'agira en ce sens de dédoubler la personnalité, de susciter une sorte d'ambivalence bien ordonnancée où le sujet se rend capable, tout en gardant conscience de sa propre identité en tant que destinataire d'un message, de poser comme extérieure à soi cette même identité en tant que productrice.

Cet enjeu nous conduit à repenser une vieille dichotomie de la théorie de la communication: celle qui oppose le locuteur à l'auditeur, l'émetteur au récepteur, le destinateur au destinataire. Rappelons d'emblée que les insuffisances de cette dichotomie ont été maintes fois démontrées, notamment par les théories systémiques et inférentielles de la communication ${ }^{1}$. Notre propos n'est pas de livrer un combat d'arrière-garde, mais de nous servir de propriétés d'une opposition qui, justement vouée aux gémonies par l'intellect de savants analystes, n'en demeure pas moins structurante de l'expérience vécue spontanée des protagonistes de la situation de communication que vise précisément notre approche phénoménologique, et s'avère en ce sens susceptible de servir de tremplin aux aspirations émotionnelles des adeptes du charisme religieux.

\section{Le vécu des positions dans la situation de communication}

Tâchons dans un premier temps de déterminer les formes phénoménologiques, c'est-à-dire les configurations, les articulations entre elles d'expériences vécues de la situation communicationnelle, qui induisent ou tendent à induire chez un protagoniste l'instauration subjective de soi comme producteur d'un discours, d'un comportement, d'une pensée. Ce vécu producteur se reconnaît, nous semble-t-il, à une forme particulière de rapport au présent, où celui-ci est perçu comme tension entre un passé et l'anticipation projective d'un avenir. Un

1 Cf. Jean-Pierre Meunier, "Nouveaux modèles de communication, nouvelles questions", in Recherches Sociologiques, XXI, n³, 1990, p. 267-288. 
acteur se sent producteur quand il dirige le produit inachevé vers un projet d'achèvement ${ }^{1}$. Du point de vue discursif, la production consiste donc en une installation de l'acte verbal dans une temporalité orientée vers un projet communicationnel. Chaque élément de discours prend sens par rapport à ce projet orientateur. Bien entendu, ce projet ne gouverne pas seul l'acte de production: cette production peut en effet développer des logiques propres de déploiement, susciter l'émergence d'un projet alternatif ou modifier le projet initial. Mais là n'est pas la question: ce qu'il nous importe de caractériser ici, c'est le vécu de la situation typique de production. Quand le discours produit le projet, ce vécu ne peut être maintenu que par l'assimilation du procès discursif au processus cognitif qui préside à la confection du projet communicationnel - «il pense à haute voix». Autrement, le vécu est celui de la réception².

L'élément qui distingue le vécu de la production de celui de la consommation, de la réception, peut en ce sens être cherché dans l'absence relative de surprise quant au contenu du discours, ou l'attribution spontanée de cette surprise à la sphère cognitive plutôt qu'à des phénomènes communicationnels. A contrario, l'acteur se définira comme récepteur quand le discours auquel il est confronté lui apparaît surprenant, ou à tout le moins quand il n'en oriente pas les composantes présentes vers un projet. Dans la situation de récepteur, le projet se dévoile par le discours, il est re-constitué dans et par l'acte communicationnel.

1 Cette conception est à rapprocher de celle qu'expose Schutz : "Lorsqu'en parlant il articule sa pensée en phases, le communicateur ne fait pas seulement l'expérience de ce qu'il prononce effectivement; dans son courant de conscience, un mécanisme compliqué de rétentions et d'anticipations relie chaque élément de sa parole à ce qui précède et à ce qui suivra, jusqu'à former l'unité de pensée à transmettre. Toutes ces expériences appartiennent à son temps intérieur" (Alfred ScHUTZ, Le chercheur et le quotidien, Paris, Méridiens Klincksieck, 1987, p. 116-117).

2 Il est abusif de ramener cette distinction à celle qui oppose traditionnellement le discours au récit, dans la mesure où il ne s'agit pas ici de rendre compte de caractéristiques objectives mais bien d'appréhension subjective ponctuelle des paroles prononcées. De plus, le niveau de structuration de la pensée au moment de l'énonciation n'est pas celui du récit: il s'agit simplement d'une «polarisation» de chacun des éléments verbaux ou idéels vers un aboutissement plus ou moins diffusément appréhendé au moment même de leur énonciation, ou en d'autres termes d'une valence signitive immédiate et labile de chaque point d'énonciation, chaque acte de parole. 


\section{Production de ces ressentir positionnels}

L'enjeu -introduire la surprise dans le discours- étant ainsi défini, examinons comment il est possible de l'atteindre par certaines formes de fusions groupales en interprétant la situation d'un groupe de prière se réclamant du Renouveau Charismatique ${ }^{1}$. D'emblée, nous distinguerons deux naissances du discours, étroitement enchâssées: la naissance individuelle et la naissance groupale.

Dans une première phase, deux aspects de la socialisation des membres du groupe doivent être pris en considération: l'intériorisation d'un lexique et celle d'une syntaxe automatique. Ces deux préalables au charisme peuvent être pris en charge par le mouvement au cours d'une période de socialisation tertiaire, d'initiation et d'apprentissage des techniques minimales requises. Pour bien prier, il faut quand même un certain entraînement (Gaëtan). Cependant, dans le cas qui nous occupe, l'essentiel de cette instruction est déjà réalisé par la société globale, les adeptes du Renouveau Charismatique provenant pour la plupart de sphères engagées dans la religion instituée. En effet, le lexique utilisé ne diffère guère de celui diffusé par l'Église catholique post-conciliaire, et peut dans ce sens s'inspirer du vocabulaire typique des girons chrétiens militants ${ }^{2}$. A noter toutefois que, la raison d'être du mouvement étant d'opposer à une conception intellectuelle ou gnosticiste de la religion un vécu intense et émotionnel de la foi, préférence sera accordée aux termes simples, spontanés, enfantins, contre le vocabulaire abstrus ou ésotérique. Dieu aime que nous nous adressions à lui avec des mots d'enfants (Jacques). De plus, l'effet d'érosion de formules répétées mécaniquement dans les offices, l'émancipation progressive des signifiants que tend à favoriser le caractère intangible des signifiés correspondants, est en partie compensé par l'insistance, l'enjouement, les gestes, bref, les artifices de persuasion et d'auto-persuasion qui accompagnent le recours à ce

1 Les situations dont il est question dans cette analyse ne concernent donc pas la démarche charismatique dans son entier. L'objectif visé ici n'est pas, en effet, de rendre compte exhaustivement des pratiques de ces croyants, mais de construire, à partir d'observations, une hypothèse généralisable sur le caractère social de toute évidence.

2 Parce que cette problématique ne nous concerne qu'indirectement ici, nous n'aborderons pas le problème des relations des communautés avec l'Église. On se reportera à l'article de Martine COHEN: "Vers de nouveaux rapports avec l'institution ecclésiastique -l'exemple du Renouveau Charismatique en France", in Archives des sciences sociales des religions, $\mathrm{n}^{\circ} 2,1985$, p. 61-79. 
lexique dans de multiples circonstances. Quant à la syntaxe, il n'est sans doute pas exagéré de la comparer à celle que manipulent plus ou moins consciemment les praticiens de la communication religieuse, les célébrants au cours de leurs offices liturgiques. Nous la qualifions d'automatique dans le sens où elle repose surtout sur une logique d'association des termes entre eux, puisée dans ces termes eux-mêmes. En d'autres termes, la syntaxe que doivent assimiler et pratiquer les intervenants associe à chaque terme un éventail de conséquents possibles, avec divers degrés de vraisemblance. Le discours peut se construire de lui-même, par échos, suites spontanées, flots de paroles. A la différence des messes cependant, chaque acteur peut exercer ce lexique et cette syntaxe ${ }^{1}$. De plus, l'abstraction et l'hiératisme étant abolis, de nombreux éléments emphatiques, métalinguistiques, ou simplement des tics verbaux peuvent apparaître ${ }^{2}$.

Une fois acquis et intériorisés ces préalables avec une assurance suffisante -généralement, ce sont les mêmes personnes qui prennent la parole d'une réunion à l'autre-, il peut se produire ce que nous nommerons une compulsion à l'expression d'un projet minimal. Nous entendons par là qu'au cours de l'effusion, une idée, une intuition peut germer dans le chef d'un des participants et le pousser à prendre la parole, sans qu'un plan précis ait été pré-établi. Le processus diffère peu, dira-t-on, de celui qui préside à la prise de parole dans un groupe quelconque. Sans doute, mais les structures spécifiques dans lesquelles il se développe ici permettent, sans que sanction groupale s'ensuive, l'expression directe de projets particulièrement flous, chaotiques, émotifs, laborieux. C'est donc une distinction de niveau qui nous conduit à parler d'un projet minimal. En l'occurrence, celui-

1 "Le langage courant comporte un certain ésotérisme. Il fait appel d'une part à des formules stéréotypées, à des notions et concepts fort classiques en milieu chrétien et, d'autre part, insère dans ces formulations des locutions plus typiques du RC: «gloire», «louange», «merci Seigneur», «guérison», «libération», «baptême ou effusion dans ou de l'Esprit», «charisme», etc. Tel quel ce langage présente la pauvreté formelle et de fond de tous les néo-clacissismes. On n'a pas souvent l'impression que les adhérents du RC s'efforcent effectivement de pénétrer au-delà des significations stéréotypées, souvent désuètes, de ces redondances de l'ancien et du rénové." (Albert DouTrelouX et al., op. cit., p. 48).

2 Cela donne des interventions du type (j'invente): “Je voudrais laisser parler mon cœur, pour dire que je suis vraiment très heureuse d'être ici avec vous, car Jésus nous dit qu'il est là, qu'il est vraiment là au milieu de nous dans toute sa gloire, c'est incroyable, et que nous devons nous réjouir et lui rendre louange pour sa présence parce qu'il nous aime, et parce que nous avons confiance en son amour plus fort que tout $(. . .)^{\prime \prime}$. 
ci prend forme dans le flux des échanges du groupe. Il peut puiser sa substance dans une réflexion antérieure à la réunion (par exemple, allusion à un événement de la semaine), s'inspirer du discours d'un autre membre, d'un chant, d'un passage d'Évangile, voire être simplement suscité par l'ambiance.

Quoi qu'il en soit, l'application au projet minimal de la syntaxe automatique via la sélection du champ lexical et cognitif adéquat permet au discours de se déployer et de surprendre. La conjonction d'un lexique et d'une syntaxe automatique ne suffisent donc pas: il faut encore que soit désignée une portion du bagage global de mots, d'idées, de poncifs, d'expressions qui différencie les prises de parole les unes des autres; et cette désignation est opérée par le projet communicationnel minimal. Ces trois conditions étant réunies, il est probable que l'intervention prenne corps sous la forme d'une consécution plus ou moins compréhensible, où le sujet s'épanche sans contrainte ressentie dans la foi.

On aura compris que l'étape du déploiement discursif, avec ses rétroactions possibles sur le projet initial, peut être considérée comme un des lieux par excellence de production de la surprise -principalement chez le locuteur-dont nous avons précisé l'enjeu précédemment. Elle n'est pas le seul, cependant, et cela s'explique par le contrôle strict auquel est soumise l'expression. Voyons en quoi consiste ce contrôle, et comment il réserve une seconde surprise au locuteur.

Un membre nous confiait, au retour d'une réunion de prière, sa joie d'avoir pu découvrir, dans les groupes de prière du Renouveau Charismatique, une structure très informelle, absolument pas dirigiste, très libre. Il est un fait que la tolérance est de mise, en accord avec les interprétations modernes de l'Évangile, et qu'elle s'impose même avec quelque ostentation; la liberté est un droit précieux, et si certains ne la goûtent pas vraiment en se détournant de Dieu, la compréhension, la prière, l'amour constituent de bien meilleures solutions que la haine et le rejet. Cependant, de nombreuses publications insistent sur l'indéniable structure de domination, sur la logique larvée de pouvoir, sur les mécanismes d'influence qui animent le groupe charismatique. De nombreux indices nous incitent à adhérer à ce point de vue; nous n'estimons pas nécessaire de les développer ici ${ }^{1}$. Il découle de ces

1 "We have already noticed that one of the roles of the pastoral team and other senior members was to "confirm" their bethren. It was also pointed out that the most 
deux remarques que, si pouvoir il y a, celui-ci doit être masqué, voilé. D'autres facteurs de contrôle du déploiement du discours, indices de -ou succédanés à- ces logiques, devraient dès lors pouvoir être mentionnés. Relevons ainsi les limites déjà abordées qu'imposent le champ lexical et la syntaxe automatique. Les valeurs intériorisées par les membres du groupe, les références à la Bible ou aux Évangiles, le contrôle social manifesté par des exclamations de louanges chuchotées pendant les prophéties par les auditeurs, peuvent être décrites également comme autant de canalisateurs du discours. La dévalorisation explicite du discours critique, perçu comme conséquente de la non-libération, comme indice de méfiance, a également pour effet le rejet immédiat de tout une série de thèmes. Certaines interventions, tournées en prières ou en prophéties, peuvent à ce propos comporter des messages comminatoires au groupe dans son ensemble:

"Tout ce qui est autour de nous est le fruit de la Sagesse de Dieu. Nous devons nous montrer reconnaissants envers Dieu pour tant de sagesse. Quand nous ronchonnons, quand nous critiquons ce qui se passe autour de nous, c'est la sagesse de Dieu que nous critiquons. Quand nous râlons sur nos voisins, sur nos collègues, quand nous médisons, c'est toujours la sagesse de Dieu qui est visée. Même quand nous critiquons les politiciens (sourires), et surtout... quand nous critiquons l'Église, c'est la sagesse de Dieu que nous critiquons. Nous devons apprendre à ne plus critiquer, mais à voir en tout l'expression de la sagesse de Dieu." (André).

Paradoxalement, ces multiples contrôles ont pour effet de rendre possible une seconde surprise: celle qui émerge de la décantation éventuelle, par le locuteur, de l'apparente logomachie qu'il a produite, en un projet explicite, différent au moins en précision, souvent en

important confirmation was that of the charismatic priest who may emphasize the "great theological insights" or the "wealth of spiritual experience" contained in the sharings or prophecies of a given member. Moreover, the topic of the discussion to be treated at the business meeting is often presented by the priest himself, with clair clues about what would be the desirable outcome. At times the few comments at the end of the meeting become an improvised homily to reject some ideas that have been expressed. Usually the priest sides with the more conservative or restrictive interpretations of the Catholic teaching (on authority, birthcontrol, abortion, ecumenical matters) which emphasize submission rather than initiative. He often presents his position as the teaching of the curch, which excludes questioning. Moreover, his heavy duties make him a somewhat remote authority figure, being still called "Rather" reverentially after five years of weekly or bi-weekly prayer meetings" (Pierre HEGY, op. cit., p. 17). 
signification, du projet minimal initial qu'il avait vaguement pressenti au moment de la compulsion à l'expression. En effet, le carcan invisible dans lequel se développe le discours conforme, polit, configure ce dernier en un message, non seulement neuf et sensé, mais de plus fondamentalement congruent avec les valeurs et attentes du groupe. Cette surprise au second degré diffère de la première en ce qu'elle est accessible en tant que telle aux autres membres du groupe (le ressentipercepteur du message n'étant pas surprenant pour ceux qui écoutent le locuteur).

S'amorce avec cette seconde surprise la naissance groupale du discours. Celle-ci se fonde en premier lieu sur une préconnaissance commune: celle de la phénoménologie de l'intervention dont nous venons de formaliser le fonctionnement. Autrement dit, l'expérience intime de réceptivité dans l'émission qui accompagne la prise de parole charismatique acquiert toute sa force persuasive quand elle est, par empathie, reconnue comme telle par les autres membres du groupe. Dépouillée de toute intentionnalité liée au locuteur, la parole ainsi prononcée s'arrache du champ cognitif, rationnel, construit, dont nous avons remarqué plus haut qu'il offre une explication alternative plausible, sans ouverture au transcendant, à la production du projet par le discours. Cette «sympathie» par laquelle chacun intuitionne profondément l'absence d'intentionnalité, la passivité réceptive de celui qui n'a que l'apparence du producteur du discours, ne peut provenir que du souvenir par chacun de cette expérience. Avoir vécu la situation paradoxale de la transe (le mot est fort dans le cas présent) permet d'admettre celle d'autrui. Nous nuancerons toutefois cette assertion en observant qu'avoir simplement approché cet état peut suffire, à condition que l'autre soit perçu comme digne de crédit.

En second lieu, la naissance groupale du message prend pour fondement les singularités des membres, sans lesquelles aucune surprise n'est concevable. Même si une redondance extrême est admise, selon le principe qui dit que la parole de Dieu n'est pas épuisable, le vécu intense d'une communication exige en effet un minimum de transfert informationnel, d'innovation. Les caractéristiques biographiques, philosophiques... de chacun, en personnalisant les projets initiaux, introduisent ce quota de nouveauté, qui fait du dire un communiquer. Troisièmement, et complémentairement, le message est intégré dans l'effusion du groupe à condition qu'il s'adresse à des connaissances, à des valeurs, à des jugements acceptables pour les autres membres. Cet évitement de l'héréticité est rendu possible et 
probable du fait de l'histoire semblable que partagent les adeptes du Renouveau Charismatique.

Ces conditions d'entrée du discours dans la logique groupale remplies, celui-ci peut être collectivement modelé de manière à poursuivre le processus de surprise. La perception sélective est le premier modelage -la première adultération?- observable. Qu'il y ait ou non eu décantation du discours par le locuteur placé en position objectivante, mais surtout si celle-ci n'a pas eu lieu, seuls les éléments les plus frappants, les plus «convenables» de l'intervention sont retenus. Cette sélection est rendue souvent indispensable par le caractère battologique et allégorique du discours produit. Elle permet aussi et surtout d'orienter dans le flux global le dire polysémique de l'individu. Elle se produit d'ailleurs aussi bien pour les prophéties que pour les lectures d'Évangile, les témoignages ou les réflexions.

Le second traitement effectué quasi spontanément est l'interprétation large. Les éléments retenus, explicités par l'un ou l'autre membre, font l'objet d'exégèses, d'inférences, de comparaisons, de compréhensions diverses. Parfois même, plusieurs interprétations se côtoient, concurremment ou complémentairement. Il arrive également qu'une de ces interprétations accommodatrices ne soit pas approuvée, moins parce qu'elle semble trahir le message que parce qu'elle ne permet pas de l'intégrer dans le flux. Diverses voies peuvent être empruntées par cette herméneutique libre, qui mènent toutes à l'alignement du «bruit» individuel dans la continuité groupale, quitte à busquer et brusquer le sens initial. La confirmation en est une, par laquelle un membre affirme que l'intervention répond précisément à une question qui le tourmentait, ou correspond exactement à ce qu'il tentait d'exprimer, ou encore ouvre ses yeux à un aspect des choses qui lui faisait gravement défaut. Cette confirmation, qui joue un rôle important dans le phénomène d'attribution dont nous parlerons plus bas, contient généralement des interprétations implicites du projet communicationnel du locuteur; or, les confirmations sont souvent le fait de membres influents du groupe, et, en tout état de cause, leur poids dépend du statut de la personne qui avalise de la sorte. Si on suppose que les membres prédominants sont, dans ce genre de situations, ceux qui ont intériorisé le plus profondément les valeurs et normes du groupe, il est permis de voir dans ce rituel une forme de contrôle social. Une autre sorte d'interprétation consiste à lier le projet décanté à des passages bibliques ou à des chants. 
Cette phase du traitement du discours induit, nous l'avons vu, un contrôle a posteriori du groupe sur ce qui se dit, distinct du contrôle $a$ priori qu'imposent les divers carcans sociaux au moment même de l'acte expressif. Ce contrôle second est extrêmement actif, puisqu'il peut aboutir à ce que le locuteur soit littéralement dépouillé de son projet communicationnel, trahi par le groupe. Il n'est cependant jamais arrivé, au cours de l'observation participante à laquelle nous nous référons ici, qu'un locuteur réfute une interprétation fournie. Une explication peut en être trouvée dans le fait que celui-ci se ressent comme étranger à son propre dire, et par conséquent démuni de tout privilège quant à la compréhension fine de sa signification.

En même temps, ce contrôle de chacun par tous peut être vu comme le fonctionnement même des prières collectives, puisque l'interprétation peut être assimilée à une compulsion à l'expression telle que celle dont nous avons parlé plus haut. Comme dans un «brainstorming», la communication peut ainsi s'engager dans un processus d'auto-alimentation soigneusement canalisé. Remarquons qu'à ce système correspond un vécu positif lié à la proximité structurale ressentie par les membres entre leur situation et l'expérience de fusion qui gît et agit dans leur inconscient. Sans entrer dans les détails de ces phénomènes sassés et ressassés par les courants psychanalytiques de la psycho-sociologie des groupes, nous noterons que la nature même de cette expérience prédispose la personne qui la vit à y découvrir une dignité totale et merveilleuse très comparable à celle qu'on attendrait d'une rencontre avec l'absolu. Cependant, et contrairement à ce qu'affirment les lieux communs sur les mouvements charismatiques, les moments de réelle fusion sont relativement peu fréquents et n'atteignent pas, à notre connaissance, les sommets mystiques dont fait bonne chair la représentation sociale. Persiste toujours une sage distance au rôle, une retenue qui permet aux contenus de garder une importance non négligeable. Certes, notre position ambiguë dans le groupe, propice à la distanciation et à l'objectivation, a pu nous désimpliquer inconsciemment de situations mystiques, fusionnelles, mais certains indices nous incitent à croire que ce biais n'explique pas seul la -relative- retenue du groupe ${ }^{1}$. En ce qui nous concerne, il semble

1 Ainsi, il arrive fréquemment que certains échangent quelque commentaire, voire plaisanterie, au cours des prières; quand un intervenant bafouille ou s'embrouille, les sourires indulgents et les réactions d'assistance témoignent également d'une certaine «sagesse» démystifiée; de même, lorsqu'un jour, un chant entamé quelques tons «trop haut», avec pour conséquences égosillements, fausses notes et 
pertinent de situer l'expérience de fusion dans l'après de ce à quoi elle se rapporte. En d'autres termes, les situations fusionnelles sont le plus souvent des reconstructions a posteriori, émises quand ont disparu les contraintes fonctionnelles qui mobilisent l'attention du moment. Ceci vaudrait tant pour les réunions de prière que pour les pèlerinages, récollections et rassemblements de plus grande ampleur dont il est régulièrement fait mention dans les groupes comme de moments forts de la foi chrétienne.

Après quelques itérations de ce processus récursif de fécondation mutuelle de la spiritualité, il arrive qu'un membre du staff articule entre elles les interventions pour en démonter et démontrer la continuité et la logique profonde. C'est alors que se produit la troisième surprise: issus en apparence de l'anarchie émotionnelle des imaginaires particuliers aux membres, des sensibilités individuelles, des préoccupations inconscientes et inavouées de chacun, les messages offrent "à qui sait les comprendre" une intelligibilité globale plus profonde encore que celle décantée auparavant. Inutile de préciser la part importante de ré-interprétation, de distorsion que requiert cet exercice: nous ne reviendrons pas sur les mécanismes qui y président, et dont nous avons montré plus haut l'efficacité. Remarquons en revanche que l'enjeu de cette nouvelle synthèse des interventions réside à nouveau dans la génération des conditions qui induisent l'acteur à se ressentir, à s'éprouver comme consommateur d'un message orientế par un projet établi par une instance extérieure, invisible et manifestement toute-puissante ${ }^{1}$. Les multiples phénomènes de canalisation, d'élagage, de contrôle préalables renforcent l'impression d'une «collusion métempirique» dans la mesure où ils réduisent l'écart entre le sens obvie et l'exégèse subtile à laquelle se livre la personne qui entreprend un tel travail de synthèse.

finalement faillite en une lamentable cacophonie, suscita un irrépressible fou rire de quelques-uns, les sourires bienveillants et amusés de l'assistance indiquèrent une décontraction, une spontanéité qu'on ne peut attendre d'un groupe centré exclusivement sur son expérience extatique intense; de nombreuses autres anecdotes du genre pourraient encore être citées.

1 Cette attribution, sur laquelle nous reviendrons ultérieurement, de l'harmonie groupale, se retrouve même dans la gestion quotidienne du groupe et de la communauté: "One of the indicators, to those engaged in discerning, that an idea's time has come or that a proposed cause of action has the blessing of the Lord is the effect they have on participants. If they create personal or collective discord, then they probably are not from the Lord" (Ralph LANE, "The Catholic Charismatic Renewal Movement in the United States: A Reconsideration", in Social Compass, XXV, 1978/1, p. 28). 
Essentiellement répétitive, circulaire, la pratique des groupes de prière se vit néanmoins sur le mode de l'historicité qui convient à une relation interpersonnelle. Cette méthodologie est à rapprocher de celle de l'Église, telle que la décrit le Père Dumont, un des personnages centraux du groupe auquel nous nous référons ici, dans un enseignement exceptionnel ${ }^{1}$ dispensé au cours de l'Avent: il s'agit de concilier une cyclicité à une progression, comme une vrille qui, dans la répétition, la circularité, parvient à avancer vers son but. Cette insertion dans l'histoire est importante du point de vue de la foi chrétienne, car le temps, créé par Dieu, indique la perfectibilité, la progression possible de l'Homme vers Dieu et l'Apocalypse. Nous ne nous appesantirons pas sur cet aspect important du vécu de la situation temporelle des groupes de prière comme «pèlerins», «acteurs en chemin vers un ailleurs». Remarquons toutefois que cette découverte de la dimension historique enfante une quatrième surprise: celle qui s'impose quand, au-delà de l'intelligibilité intrinsèque du discours, de sa cohérence axiologique et informationnelle, de sa transversalité, de sa continuité interpersonnelle, émerge l'évidence de son orientation temporelle à long terme, vers un projet puissant et inconnu que l'improviste et la labilité des discours initiaux étaient à mille lieues d'imaginer. Cette grande fresque historique, qu'ébauchent presqu'exclusivement les membres influents du groupe, met en œuvre ces mêmes phénomènes de perception sélective, d'interprétation libre, de canalisation dont nous avons déjà souligné le rôle essentiel.

\section{Effets de ces quatre surprises}

Les divers mécanismes que nous avons inférés par empathie et observation ont tous pour effet de confronter les membres du groupe à un projet à la fois séduisant et inattendu, c'est-à-dire à une synthèse fascinante par sa valeur axiologique, pratique,... et par son statut d'évidente extériorité. Cette origine autre du discours est attestée $a$ contrario seulement: le projet n'est pas conçu comme produit par les individus puisque la co-expérience de la production du discours invite chacun à induire chez chacun le statut de medium, apparemment producteur, réellement spectateur du sens émergeant; il n'est pas non plus transmis par un individu-prophète, qui en aurait l'exclusive maîtrise,

1 Car intellectuel par définition. 
puisque chacun intervient dans sa conformation progressive; pas plus n'est-il à proprement parler une production idéologique groupale, puisque le groupe dans son ensemble est interloqué par sa décantation quand elle a lieu. L'origine du message compris demeure donc mystérieuse, à moins que des logiques ne prennent en charge la fermeture de l'alternative insoluble en attribuant ce message à une instance pneumatique extérieure...

Ce processus d'attribution apparaît dans ce que nous appellerons les «passages au discours méta», c'est-à-dire les recours à point nommé à une explication implicite ou explicitement formulée de ces «étranges coïncidences». C'est ainsi qu'une personne du staff peut se livrer à des précisions, adressées aux néophytes surtout, sur la franchise des participants, la signification de leur conduite, l'absence de concertation dans les préparations.

Mais, bien au-delà de cette attribution explicite, destinée surtout à officialiser ce que chacun devine, ce sont d'autres logiques qui déterminent l'attribution du projet à Dieu. L'évitement, l'évidement du discours critique, intellectualisant est au fondement de ces logiques: chercher ailleurs que dans la volonté divine l'explication de ces mystères, c'est galvauder la raison que Dieu nous autorise à exercer. Il persuade, mais ne convainc pas; il fournit aux hommes tous les indices qui doivent les mener à la foi, mais nous laisse libres de ne pas entendre son appel. La foi ne s'impose pas, elle ne se déduit ni ne se réduit aux schèmes faillibles de notre raison mortelle: elle résulte d'un acte de volonté sans cesse réitéré.

Ainsi neutralisé tout discours critique, pour laisser somme toute la part belle à l'intuition, au feeling, au bon sens -à l'idéologie?-, on comprend mieux la puissance formidable des mécanismes d'inversion des vécus des situations communicationnelles: une fois acquise cette valorisation fidéiste, il suffit en effet de placer l'acteur dans une situation paradoxale où le chaos fait place au Cosmos, le désordre spontané à l'ordre spontané, pour que celui-ci puisse y découvrir les indices qui confortent sa tendance première. En d'autres termes, le résultat le plus probant des mécanismes que nous avons évoqués réside dans un renversement du maximum de vraisemblance qui guide le choix d'une personne vers une explication au détriment d'une autre. Ce renversement de l'onus probandi est produit par les surprises successives que provoque la mise en évidence de coïncidences, de régularités, d'intelligibilités contraires aux attentes que suscite une vision du monde gouverné par l'anonyme, l'inordonné, l'involontaire. Il s'agit 
donc de mettre en place des structures ordonnatrices latentes et d'assimiler l'ordre ainsi créé à une volonté anthropomorphique omnipotente, omnisciente, omniprésente, bref, divine.

Dans ce sens, la participation aux réunions de prière n'est qu'un aspect secondaire d'une Weltanschauung, pour employer un terme pompeux, transversale à tous les domaines expérientiels de la vie quotidienne. D'autres aspects des pratiques des membres du Renouveau Charismatique abondent en ce sens, notamment concernant le «partage des expériences de la semaine». Les chants en langues, caractéristique spectaculaire non négligeable du Renouveau Charismatique, peuvent être compris dans le schéma que nous avons décrit pour les prophéties et les témoignages. La seule différence notable est que le sens ne peut pas être découvert par un travail de décantation explicite: tout se passe comme si ce sens était connu de tous. Nous n'aborderons pas ce débat, bien plus large que la thématique que nous visons ici. 